\title{
Prevalence of and Trends for Dyslipidemia among Pilots from one Airline in China
}

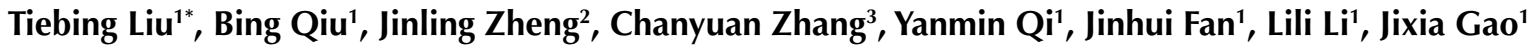 \\ ${ }^{1}$ Civil Aviation Medicine Center, Civil Aviation Administration of China (Civil Aviation General Hospital), Beijing 100123, \\ China \\ ${ }^{2}$ Air China, Southwest Branch, Chengdu 610202, China \\ ${ }^{3}$ Department of Clinical Laboratory, Civil Aviation General Hospital (Peking University Civil Aviation School of Clinical \\ Medicine), Beijing 100123, China
}

Corresponding Author: Tiebing Liu, Civil Aviation Medicine Center, Civil Aviation Administration of China (Civil Aviation General Hospital), Beijing 100123, China. Tel: +86-1085762244-2276, Email: Itbing@yeah.net

Received October 22, 2018; Accepted February 2, 2019; Online Published March 2, 2019

\begin{abstract}
Introduction: Most of previous studies on dyslipidemia among pilots were cross-sectional surveys which cannot explore the temporal trend of dyslipidemia, and these studies were normally conducted at least five years ago. In addition, studies focusing on pilots in Southwest China are scarce. This study aimed to describe the prevalence of and trends in dyslipidemia among pilots from an airline in Southwest China.

Methods: This cross-sectional study evaluated the prevalence of adverse concentrations of total cholesterol (TC), low-density lipoprotein cholesterol (LDL-C), high-density lipoprotein cholesterol (HDL-C), and triglycerides (TG) among all pilots from one airline in Southwest China in 2015. Analyses of linear trends in age-adjusted prevalence of dyslipidemia were conducted overall across a 3-year period (2013, 2014, and 2015).

Results: In 2015, 3.4\% of participating pilots had a high TC concentration, 16.4\% had a high TG concentration, 2.6\% had a high LDL-C concentration, $25.1 \%$ had a low HDL-C concentration, and 33.1\% had dyslipidemia. Between 2013 and 2015, there were decreasing linear trends in age-adjusted prevalence of high TG $(32 \%$ vs. $19.9 \% ; P<0.001)$ and dyslipidemia $(51.9 \%$ vs. $38.4 \% ; P<0.001)$. During this 3 -year period, declining trends were also observed in age-adjusted prevalence of LDL-C $(10.0 \%$ vs. $4 \% ; P<0.001)$ and $\mathrm{HDL}-\mathrm{C}$ (33.2\% vs. $28.3 \% ; P=0.015)$. However, there was no change from 2013 to 2015 in age-adjusted prevalence of high TC $(2.8 \%$ vs. $5.0 \% ; P=0.168)$. Conclusion: From 2013 to 2015, there were favorable trends in the prevalence of high TG, low HDL-C, high LDL-C, and overall dyslipidemia but no significant change in high TC among pilots from one airline in southwest China. The reasons for these trends need to be further explored.

Keywords: Dyslipidemia, Pilot, Prevalence, Trends, Lipids
\end{abstract}

Citation: Liu T, Qiu B, Zheng J, Zhang C, Qi Y, Fan J, Li L, Gao J. Prevalence of and trends for dyslipidemia among pilots from one airline in China Int J Travel Med Glob Health. 2019;7(1):18-22. doi:10.15171/ijtmgh.2019.05.

\section{Introduction}

Dyslipidemia has been associated with arthrosis and cardiovascular disease (CVD) ${ }^{1-3}$ which may cause sudden incapacitation ${ }^{4,5}$ and long-term disability as well as a loss of license among commercial pilots. ${ }^{6}$ Dyslipidemia normally refers to an adverse concentration in one or more of four lipids, including total cholesterol (TC), low-density lipoprotein cholesterol (LDL-C), triglycerides (TG), and low levels of high density lipoprotein cholesterol (HDL-C). Early identification and treatment of dyslipidemia may improve long-term health outcomes. However, due to the lack of obvious early signs, dyslipidemia tends to be overlooked.
Several studies have reported adverse lipid profiles among pilots in China. ${ }^{7-9}$ Most previous studies on dyslipidemia among pilots were cross-sectional surveys which cannot explore the temporal trend of dyslipidemia, and these studies were conducted at least five years ago. ${ }^{8,10}$ In addition, studies focusing on pilots in Southwest China are scarce. Therefore, information on the current prevalence of and trends in dyslipidemia remain limited, which might hinder the implementation of pertinent and effective control and prevention strategies.

The objectives of this study were to describe the prevalence of adverse serum concentrations of TC, LDL-C, HDL-C, and

Copyright $(\odot 2019$ The Author(s). This is an open-access article distributed under the terms of the Creative Commons Attribution License (http:// creativecommons.org/licenses/by/4.0), which permits unrestricted use, distribution, and reproduction in any medium, provided the original work is properly cited. 
TG among pilots in Southwest China in 2015 and to suggest the trends since 2013.

\section{Methods}

\section{Study Subjects}

This study was a cross-sectional study. In China, every civil pilot is required to undergo a physical examination annually. All male pilots of an airline in Chengdu, Sichuan from 2013 to 2015 were included in the study; the number of participants was 860 in 2013, 970 in 2014, and 1063 in 2015. All persons gave their informed consent prior to their inclusion in the study.

\section{Data Collection}

All data used in this study was collected by the aviation doctors from Medical Examination Database in the airline, including subjects' height, weight, TC, TG, HDL-C, and LDL-C levels. All data was anonymized before the researchers could access it.

Definitions for Adverse Lipid Concentrations and BMI The cut-off points for blood lipid indices were defined according to the Chinese guideline for the management of dyslipidemia in adults. ${ }^{11}$ Adverse lipid concentrations were defined as follows: TC concentration of $240 \mathrm{mg} / \mathrm{dL}$ or higher; HDL-C concentration lower than $40 \mathrm{mg} / \mathrm{dL}$; LDL-C concentration of $160 \mathrm{mg} / \mathrm{dL}$ or higher (to convert TC, HDL-C, and non-HDL-C to millimoles per liter, multiply by 0.0259 ); and TG concentration of $200 \mathrm{mg} / \mathrm{dL}$ or higher (to convert TG to millimoles per liter, multiply by 0.0113 ). Dyslipidemia was defined as an adverse concentration in 1 or more of the 4 lipids.

Body mass index (BMI) is calculated by dividing weight in kilograms by the square of height in meters rounded to the nearest 10th. The cut-off points for BMI were defined according to the Guidelines for Prevention and Control of Overweight and Obesity in Chinese Adults. ${ }^{12}$ Pilots were categorized as underweight (BMI $\left.<18.5 \mathrm{~kg} / \mathrm{m}^{2}\right)$, normal weight (BMI 18.5 to $23.9 \mathrm{~kg} / \mathrm{m}^{2}$ ), overweight (BMI 24 to 27.9 $\left.\mathrm{kg} / \mathrm{m}^{2}\right)$, or obese $\left(\mathrm{BMI} \geq 28 \mathrm{~kg} / \mathrm{m}^{2}\right)$.

\section{Statistical Analysis}

Prevalence estimates and 95\% CI of adverse concentrations of TC, HDL-C, LDL-C, and TG as well as dyslipidemia are reported. The study population was categorized into the following 4 age bands (in years): 20-29, 30-39, 40-49, and 5060. The percentage and its 95\% CI were calculated for 2014 . Trend analyses were based on weighted data. The percentages for pilots aged 20-60 years were age-adjusted by direct method to the projected 2010 census population using the age groups 20-29, 30-39, 40-49, and 50-60 years. Hypotheses of no survey trends in age-adjusted percentages over the 3-year period from 2013 to 2015 were tested by logistic regression analyses. Rejection of this null hypothesis implied the existence of a trend. All statistical analyses were carried out using STATA version 12.0. A two-side $P$ value of $<0.05$ was considered to be statistically significant.

\section{Results}

In 2015, among pilots aged 20 to 60 years, 3.4\% (95\% CI, $2.3 \%-4.5 \%)$ had a high TC concentration, $16.4 \%$ (95\% CI, $14.1 \%-18.6 \%)$ had a high TG concentration, $2.6 \%$ (95\% CI, $1.7 \%-3.6 \%$ ) had a high LDL-C concentration, $25.1 \%$ (95\% CI, $22.5 \%-27.7 \%$ ) had a low HDL-C concentration, and $33.1 \%$ (95\% CI, 30.3\%-35.9\%) had dyslipidemia (Table 1).

The prevalence of four types of dyslipidemia all increased with age (all $P<0.001$ ). Specifically, for high TG, low HDL-C, and dyslipidemia, the highest prevalence was observed in the age group of $50-59$ years $(26.9 \%, 7.7 \%$, and $51.9 \%$, respectively); whereas for high LDL-C and low HDL-C, the highest prevalence was found in pilots aged $40-49$ years $(6.5 \%$ and $33.1 \%$, respectively); for high LDL-C and low HDL-C, and then declined thereafter (Table 1).

The prevalence of high TG, low HDL-C, and dyslipidemia (except for high TC and high LDL-C) increased with BMI, with the highest prevalence being observed in the obese group $(29.0 \%, 46.4 \%$, and $59.4 \%$, respectively). Specifically, the prevalence of high TG climbed from $9.7 \%$ to $23.8 \%$

Table 1. Prevalence of Dyslipidemias in Pilots Aged 20 to 60 Years From Southwest China, 2015

\begin{tabular}{|c|c|c|c|c|c|c|}
\hline Characteristics & Participants, No. & TG ( $(\geq 200 \mathrm{mg} / \mathrm{dL})$ & $\mathrm{TC}(\geq 240 \mathrm{mg} / \mathrm{dL})$ & LDL-C ( $\geq 160 \mathrm{mg} / \mathrm{dL})$ & HDL-C (<40 mg/dL) & Dyslipidemia \\
\hline Total & 1063 & $16.4(14.1-18.6)$ & $3.4(2.3-4.5)$ & $2.6(1.7-3.6)$ & $25.1(22.5-27.7)$ & $33.1(30.3-35.9)$ \\
\hline \multicolumn{7}{|l|}{ Age, years } \\
\hline I , 20-29 & 550 & $10.0(7.5-12.5)$ & $1.5(0.5-2.5)$ & $0.9(0.1-1.7)$ & $19.5(16.1-22.8)$ & $25.1(21.5-28.7)$ \\
\hline II , 30-39 & 322 & $23.3(18.7-27.9)$ & $4.3(2.1-6.6)$ & $3.4(1.4-5.4)$ & $30.4(25.4-35.5)$ & $41.0(35.6-46.4)$ \\
\hline III, 40-49 & 139 & $21.6(14.7-28.4)$ & $7.2(2.9-11.5)$ & $6.5(2.4-10.6)$ & $33.1(25.3-40.9)$ & $39.6(31.4-47.7)$ \\
\hline IV , 50-59 & 52 & $26.9(14.9-39)$ & $7.7(0.4-14.9)$ & $5.8(-0.6-12.1)$ & $30.8(18.2-43.3)$ & $51.9(38.3-65.5)$ \\
\hline$P$ value for trend & & $<0.001$ & $<0.001$ & $<0.001$ & $<0.001$ & $<0.001$ \\
\hline \multicolumn{7}{|l|}{ BMI category } \\
\hline A, Normal weight & 557 & $9.7(7.2-12.2)$ & $2.7(1.3-4)$ & $2.0(0.8-3.1)$ & $14.7(11.8-17.7)$ & $21.4(18-24.8)$ \\
\hline B, Overweight & 421 & $23.8(19.7-27.8)$ & $4.3(2.3-6.2)$ & $3.1(1.4-4.7)$ & $36.1(31.5-40.7)$ & $45.4(40.6-50.1)$ \\
\hline C, Obese & 69 & $29.0(18.3-39.7)$ & $2.9(-1.1-6.9)$ & $4.3(-0.5-9.2)$ & $46.4(34.6-58.1)$ & $59.4(47.8-71)$ \\
\hline
\end{tabular}

a The trends were tested by logistic regression analyses and adjusted for age. 
to $29 \%$ for pilots in the normal, overweight, and obese categories, respectively $(P<0.001)$ Comparable estimates for the prevalence of low HDL-C were $14.7 \%, 36.1 \%$, and $46.4 \%$, respectively $(P<0.001)$ and for the prevalence of overall dyslipidemia were $21.4 \%, 45.4 \%$, and $59.4 \%$, respectively $(P<0.001)$; however, the prevalence rates of high TC and high LDL-C were not statistically different $(P=0.293$ and $P=0.141$, respectively) (Table 1 ).

Between 2013 and 2015, there were decreasing linear trends in age-adjusted prevalence of high TG [32\% (95\%CI, $28.9 \%-35.1 \%$ ) vs. $19.9 \%$ (95\% CI, $17.5 \%-22.3 \%) ; P<0.001]$ and dyslipidemia [51.9\% (95\% CI, $48.6 \%-55.2 \%)$ vs. $38.4 \%$ (95\% CI, 35.5\%-41.3\%); $P<0.001]$. During this 3-year period, declining trends were also observed in the age-adjusted prevalence of LDL-C [10.0\% (95\% CI, 8.0\%-12.0\%) vs. $4 \%$ (95\% CI, 2.9\%-5.2\%); $P<0.001]$ and HDL-C [33.2\% (95\%CI, $30 \%-36.3 \%$ ) vs. $28.3 \%$ (95\% CI, $25.6 \%-31 \%$ ); $P=0.015$ ]. There was no change from 2012 to 2014 in age-adjusted prevalence of high TC $[2.8 \%(95 \% \mathrm{CI}, 1.7 \%-3.9 \%)$ vs. $5.0 \%$ (95\% CI, 3.7\%-6.3\%); $P=0.168$ ] (Figure 1; Table 2).

Adverse lipid concentrations include TG $\geq 200 \mathrm{mg} / \mathrm{dL}$, TC $\geq 240 \mathrm{mg} / \mathrm{dL}, \mathrm{LDL}-\mathrm{C} \geq 160 \mathrm{mg} / \mathrm{dL}$, and HDL-C $<40 \mathrm{mg} / \mathrm{dL}$. Dyslipidemia was defined as an adverse concentration in 1 or more of the 4 lipids. All comparisons except TC showed a significant linear trend between 2013 and $2015(P<0.05)$. Sample sizes for TG, TC, LDL-C, HDL-C, and dyslipidemia were 860 in 2013, 970 in 2014, and 1063 in 2015. Error bars indicated 95\% CIs. All estimates are based on weighted data age-adjusted by direct method to the year 2010 population

Table 2. Trends for Dyslipidemia in Pilots aged 20 to 60 years in an Airline from 2013 to 2015

\begin{tabular}{lcccc}
\hline Factors & $\mathbf{2 0 1 3}$ & $\mathbf{2 0 1 4}$ & $\mathbf{2 0 1 5}$ & $\begin{array}{c}\boldsymbol{P} \text { Value for } \\
\text { Trend }^{\mathrm{a}}\end{array}$ \\
\hline High TG & $32(28.9-35.1)$ & $22(19.4-24.6)$ & $19.9(17.5-22.3)$ & $<0.001$ \\
High TC & $2.8(1.7-3.9)$ & $6.8(5.2-8.4)$ & $5(3.7-6.3)$ & 0.168 \\
High LDL-C & $10(8-12)$ & $11.7(9.7-13.8)$ & $4(2.9-5.2)$ & $<0.001$ \\
Low HDL-C & $33.2(30-36.3)$ & $23(20.3-25.6)$ & $28.3(25.6-31)$ & 0.015 \\
Dyslipidemia & $51.9(48.6-55.2)$ & $39.2(36.1-42.3)$ & $38.4(35.5-41.3)$ & $<0.001$ \\
\hline
\end{tabular}

aThe trends were tested by logistic regression analyses and adjusted for age. using the age groups 20-29,30-39, 40-49, and 50-60 years.

\section{Discussion}

The present study described the prevalence of adverse serum lipid levels in 2015 and trends from 2013 to 2015 among pilots from an airline in Southwest China, demonstrating that favorable trends during this 3-year period in high TG, low HDL-C, and high LDL-C were observed; an adverse lipid profile continues to be observed. In 2015, 33.1\% pilots had dyslipidemia. Specifically, $16.4 \%$ had high TG, $25.1 \%$ had low HDL-C, 3.4\% had high TC, and 2.6\% had high LDL-C. Therefore, the 2 most common dyslipidemias were low HDL-C and high TG, which was consistent with the adverse lipid pattern reported by previous studies regarding civil pilots $^{9,13-15}$ and the general population in China. ${ }^{16}$

Epidemiological studies have reported that low HDL-C is an established risk factor for coronary heart disease. ${ }^{17-19}$ Among civil pilots, one study reported a low HDL-C prevalence of $45.9 \%$ in those from Northwest China, ${ }^{9}$ whereas the corresponding figure in a recent study reported by Yu et $\mathrm{al}^{13}$ in 2015 was $29.2 \%$ in pilots aged 40 to 59 years. Among Chinese male adults in 2010, the prevalence of low HDL-C was $44.8 \%{ }^{16}$ In the current study, the prevalence was $25.1 \%$, which was significantly lower than that reported in previous studies. ${ }^{15}$ These inconsistent results may be related to different diet factors, pilot populations, study periods, or racial factors.

There is increasing evidence of a strong link between raised TG and coronary heart disease risk. ${ }^{20}$ The prevalence of high TG reported by previous studies ranged from $12.8 \%$ to $26.7 \%{ }^{9,13-15}$ Moreover, in the current study, this figure in 2014 was $16.4 \%$, which was higher than that among Chinese adults in 2010 (11.3\%). ${ }^{16}$ The reasons for higher prevalence of high TG among pilots may be because pilots tend to work under high pressure and an irregular schedule (i.e., nightshift work) and lack exercise; in addition, the diet of some pilots have high levels of oil.

Epidemiological evidence regarding the trends of adverse lipid concentrations is limited and controversial. A previous study on dyslipidemia in 305 civil pilots in China reported that increasing trends for prevalence of hyperlipidemia

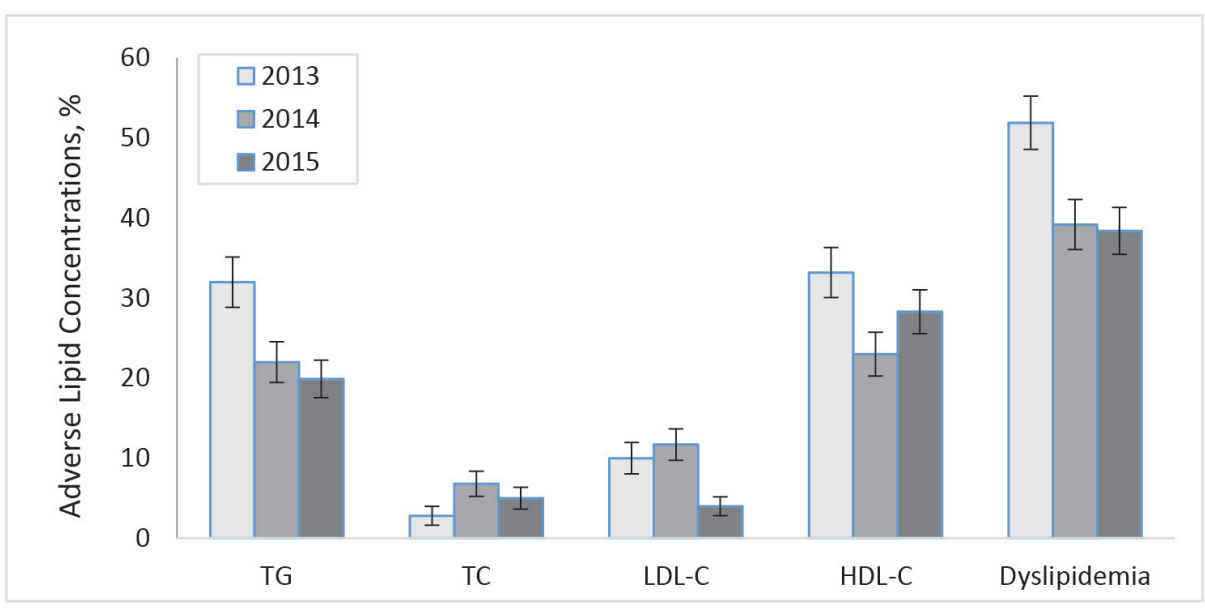

Figure 1. Prevalence of Adverse Lipid Concentrations over Time Among Pilots From Southwest China, 2013-2015. 
(including high TG, high TC, and high LDL-C) from 2006 to 2011 were observed. ${ }^{9}$ Another study reported favorable trends of the prevalence of high TC, high LDL-C, and low HDL-C between 2007 and 2008. ${ }^{10}$ These inconsistent results might be attributed to differences in pilot population, study period, racial factors, or sample sizes. The current study found a favorable trend in the prevalence of adverse serum lipid concentrations among pilots in Southwest China from 2013 to 2015. Specifically, the declines reported herein included the prevalence of high TG, low HDL-C, high LDL-C, and overall dyslipidemia, which were similar to the results reported by a previous study. ${ }^{10}$

The association between BMI and serum lipid concentrations has been previously described. ${ }^{21,22}$ In this analysis, the higher prevalence of high TG, low HDL-C, and dyslipidemia increased with age with the highest prevalence in obese pilots, which was similar to most previous studies. ${ }^{10,23,24}$ However, the prevalence of high TC and LDL-C were not statistically different between BMI groups. This was inconsistent with several studies. ${ }^{24}$ One possible explanation for these seemingly paradoxical findings is that serum levels TC and LDL-C are linked to a variety of factors other than BMI, i.e., diet ${ }^{25}$ and physical activity. ${ }^{26}$ The relevant reasons need to be further explored.

There may be differences in the distribution of abnormal lipid types among pilots in different fields and periods, suggesting that corresponding prevention and control strategies should be formulated according to the specific epidemic characteristics of abnormal lipids in pilots, so as to achieve the purpose of improving the prevention and control effects. To strengthen the prevention and treatment of dyslipidemia in civil aviation pilots, in addition to regular blood lipid examinations, it is also suggested that the aviation field strengthen the popularization of blood lipid health knowledge and actively advocate a healthy lifestyle, including a reasonable diet and physical exercise. At the same time, pilots with dyslipidemia should be treated actively, and the health level of pilots should be improved through comprehensive measures, which is of great significance for ensuring flight safety and prolonging flight life.

Several limitations in the present study should be considered. First, it should be noted that this study only targeted pilots from one airline based in Southwest China. Therefore, data from other pilot populations was needed to confirm the favorable trends. Second, the number of civil pilots included in the current study was less than that of several large-scale surveys on relevant topics among the general population; nevertheless, the target population of this study is much smaller. Third, the present study period was relatively short, including only 3 years. Nevertheless, there might be sufficient evidence to suggest the prevalence of and trends in dyslipidemia among pilots in the airline. Further study with more pilots from other regions of China and a longer followup period will provide more insight into the epidemiology of adverse lipid levels among civil pilots in China.

\section{Conclusion}

Among pilots aged 20-60 years from one airline based in

\section{Research Highlights}

\section{What Is Already Known?}

Little is known about the prevalence of and trends in dyslipidemia among pilots from an airline in Southwest China.

\section{What This Study Adds?}

- Early identification and treatment of dyslipidemia may improve long-term health outcomes.

- Among the pilots in this study, $33.1 \%$ had overall dyslipidemia, and the 2 most common dyslipidemias were low HDL-C (25.1\%) and high TG (16.4\%).

- From 2013 to 2015, there were favorable trends in the prevalence of high TG, low HDL-C, high LDL-C, and overall dyslipidemia but no significant change in high TC.

- Corresponding prevention and control strategies should be formulated according to the specific epidemic characteristics of abnormal lipids in pilots, so as to achieve the purpose of improving the prevention and control effects.

- Pilots with dyslipidemia should be treated actively, and the health of pilots should be improved through comprehensive measures, which is of great significance for ensuring flight safety and prolonging flight life.

Southwest China in 2015, 33.1\% had overall dyslipidemia. The 2 most common dyslipidemias were low HDL-C (25.1\%) and high TG (16.4\%). From 2013 to 2015, there were favorable trends in the prevalence of high TG, low HDL-C, high LDL-C, and overall dyslipidemia but no significant change in high TC. The reasons for these trends need to be further explored.

\section{Authors' Contributions}

Study concept and design: TL, BQ, YQ. Analysis and interpretation of data: TL, BQ, JZ. Drafting of the manuscript: TL, JF, LL, JG. Critical revision of the manuscript for important intellectual content: $\mathrm{BQ}$. Revise the manuscript: CZ. Statistical analysis: TL. TL had full access to all of the data in the study and takes responsibility for the integrity of the data and the accuracy of the data analysis.

\section{Conflict of Interest Disclosures}

The authors declare that there is no conflict of interest regarding the publication of this paper.

\section{Ethical Approval}

The study protocol was reviewed and approved by the Ethics Committee of the Civil Aviation General Hospital (CAMC00001652-015) and was performed in accordance with the ethical standards laid out in the 1964 Declaration of Helsinki.

\section{Funding/Support}

This study was supported by the National Natural Science Fund Committee and Civil Aviation Administration of China Jointly Funded Project (No. C0023193) and the Civil Aviation 
Medicine Center (General Hospital) Scientific Research Fund-funded Project (No. 201939).

\section{Acknowledgments}

The authors are grateful to the anonymous reviewers for their helpful comments and valuable suggestions and for critically reviewing the paper.

\section{References}

1. Kit BK, Kuklina E, Carroll MD, Ostchega Y, Freedman DS, Ogden $\mathrm{CL}$. Prevalence of and trends in dyslipidemia and blood pressure among US children and adolescents, 1999-2012. JAMA Pediatr. 2015;169(3):272-279. doi:10.1001/jamapediatrics.2014.3216.

2. Stamler J, Wentworth D, Neaton JD. Is relationship between serum cholesterol and risk of premature death from coronary heart disease continuous and graded? Findings in 356,222 primary screenees of the Multiple Risk Factor Intervention Trial (MRFIT). JAMA. 1986;256(20):2823-2828. doi:10.1001/ jama.1986.03380200061022.

3. Assmann G, Schulte H, Funke H, von Eckardstein A. The emergence of triglycerides as a significant independent risk factor in coronary artery disease. Eur Heart J. 1998;19 Suppl M:M8-14.

4. Bennett G. Pilot incapacitation and aircraft accidents. Eur Heart J. 1988;9 Suppl G:21-24. doi:10.1093/eurheartj/9.suppl_G.21.

5. Ady Wirawan IM, Griffiths RF, Larsen PD. Cardiovascular Tests for Risk Assessment in Asymptomatic Adults and Implications for Pilots. Aerosp Med Hum Perform. 2018;89(7):648-656. doi:10.3357/amhp.5065.2018.

6. Ekstrand K, Bostrom PA, Arborelius M, Nilsson JA, Lindell SE. Cardiovascular risk factors in commercial flight aircrew officers compared with those in the general population. Angiology. 1996;47(11):1089-1094. doi:10.1177/000331979604701109.

7. Lou F, Shen Q, Xu L, et al. Prevalence of Hypertension and Associated Risk Factors among Civil Aviation Pilots. Journal of Environmental \& Occupational Medicine. 2015;32(8):735-738. [Chinese].

8. Fei $X, X u Z M, L i Q$, et al. The survey and analysis of the blood lipid status of civil airlines pilots. Zhonghua Hang Kong Hang Tian Yi Xue Za Zhi. 2012;23(4):245-249.

9. Zhao R, Xiao D, Fan X, et al. Epidemiological survey of dyslipidemia in civil aviators in china from 2006 to 2011. Int J Endocrinol. 2014;2014:215076. doi:10.1155/2014/215076.

10. Shen Y, Nong QL, Song L, et al. Survey of the blood lipid level of 192 civil aviators. Zhonghua Hang Kong Hang Tian Yi Xue Za Zhi. 2012;23(1):58-60.

11. Joint Committee for the Revision of the Guidelines for Prevention and Treatment of Dyslipidemia. Guidelines for the prevention and treatment of dyslipidemia in Chinese adults (revised 2016). Chinese Circulation Journal. 2016;31(10):7-28.

12. Chen C, Lu FC. Guidelines for Prevention and Control of Overweight and Obesity in Chinese Adults. Acta Nutrimenta Sinica. 2004;26(1):1-4.
13. Yu H, Liu G, Liu T, et al. Distribution and aggregation analysis of biological risk factors for cardiovascular disease among 831 pilots over 40 years old. Zhongguo Shi Yong Nei Ke Za Zhi. 2015;35(S1):5-7. [Chinese].

14. Zhao $R$, Fan $X$, Dong $Y$, et al. A survey and investigation on the blood lipid levels of Northwest China's civil aviation pilot. Zhongguo Yi Yao Dao Bao. 2014;11(5):116-119. [Chinese].

15. Liu AB, Wang QQ, Zhou XJ, et al. Blood lipid profile survey of military pilots. Zhonghua Hang Kong Hang Tian Yi Xue Za Zhi. 2005;16(4):280-287.

16. Li T, Zhang Y, Zhu Y. Analysis on the dyslipidemia in civil pilots. Zhonghua Hang Kong Hang Tian Yi Xue Za Zhi. 2012;23(3):204207.

17. Gordon DJ, Probstfield JL, Garrison RJ, et al. High-density lipoprotein cholesterol and cardiovascular disease. Four prospective American studies. Circulation. 1989;79(1):8-15. doi:10.1161/01.CIR.79.1.8.

18. Castelli WP, Doyle JT, Gordon T, et al. HDL cholesterol and other lipids in coronary heart disease. The cooperative lipoprotein phenotyping study. Circulation. 1977;55(5):767-772. doi:10.1161/01.CIR.55.5.767.

19. Wilkins JT, Ning $\mathrm{H}$, Stone NJ, et al. Coronary heart disease risks associated with high levels of HDL cholesterol. J Am Heart Assoc. 2014;3(2):e000519. doi:10.1161/jaha.113.000519.

20. Nordestgaard BG, Varbo A. Triglycerides and cardiovascular disease. Lancet. 2014;384(9943):626-635. doi:10.1016/s01406736(14)61177-6.

21. Freedman DS, Goodman A, Contreras OA, DasMahapatra $\mathrm{P}$, Srinivasan SR, Berenson GS. Secular trends in BMl and blood pressure among children and adolescents: the Bogalusa Heart Study. Pediatrics. 2012;130(1):e159-166. doi:10.1542/peds.20113302.

22. Freedman DS, Dietz WH, Srinivasan SR, Berenson GS. The relation of overweight to cardiovascular risk factors among children and adolescents: the Bogalusa Heart Study. Pediatrics. 1999;103(6 Pt 1):1175-1182. doi:10.1542/peds.103.6.1175.

23. Sun GZ, Li Z, Guo L, Zhou Y, Yang HM, Sun YX. High prevalence of dyslipidemia and associated risk factors among rural Chinese adults. Lipids Health Dis. 2014;13:189. doi:10.1186/1476511x-13-189.

24. Cai L, Zhang L, Liu A, Li S, Wang P. Prevalence, awareness, treatment, and control of dyslipidemia among adults in Beijing, China. J Atheroscler Thromb. 2012;19(2):159-168. doi:10.5551/ jat. 10116

25. Welsh JA, Sharma A, Cunningham SA, Vos MB. Consumption of added sugars and indicators of cardiovascular disease risk among US adolescents. Circulation. 2011;123(3):249-257. doi:10.1161/ CIRCULATIONAHA.110.972166.

26. Thorland WG, Gilliam TB. Comparison of serum lipids between habitually high and low active pre-adolescent males. Med Sci Sports Exerc. 1981;13(5):316-321. doi:10.1249/00005768198105000-00008. 